\title{
Nuevos Reportes de Arrecifes Coralinos en el Caribe de Honduras
}

\author{
Andrés Alegría ${ }^{1}$ y Cristhian Pérez ${ }^{2}$
}

\begin{abstract}
Resumen. La exploración de los arrecifes coralinos en el Caribe hondureño ha sido enfocada principalmente en las inmediaciones de las Islas de la Bahía y de los Cayos Cochinos, mientras que mucho menos se conoce sobre la distribución, extensión y salud de estos ecosistemas a lo largo de la plataforma continental dentro de los primeros $10 \mathrm{~km}$ de la costa. Sin embargo, la pesca cotidiana de especies asociadas a arrecifes (e.g. Lutjanus synagris, Ocyurus chrysurus) capturadas cerca de la costa por los pescadores artesanales es evidencia sobre la presencia de arrecifes coralinos agregados a lo largo de la plataforma continental. Tras una exploración inicial dentro y alrededor del Refugio de Vida Silvestre Barras de Cuero y Salado identificamos 15 sitios con cobertura bentónica arrecifal previamente no documentada.
\end{abstract}

Palabras clave. Áreas protegidas marinas, pesquería artesanal, Refugio de Vida Silvestre Barras de Cuero y Salado.

\section{New Reports of Coral Reefs in the Honduran Caribbean}

\begin{abstract}
The exploration of coral reefs across the Honduran Caribbean has mainly focused around the Bay Islands and Cayos Cochinos, while much less is known about the distribution, extension and health of these ecosystems along the coastal shelf within the first $10 \mathrm{~km}$ off shore. However, daily catches of reef-associated species (e.g. Lutjanus synagris, Ocyurus chrysurus) from the near coast fisheries are evidence for the presence of aggregated coral reefs throughout the continental shelf. Through an initial exploration of sites within and around the Wild Life Refuge Barras de Cuero y Salado we identified 15 sites with previously undocumented coral reef coverage.
\end{abstract}

Key words. Artisanal fisheries, Barras de Cuero y Salado Wild Life Refuge, marine protected areas,.

\section{Introducción}

La exploración de los arrecifes coralinos en Honduras ha sido considerable en las Islas de la Bahía y de los Cayos Cochinos (Bouchon et al. 2001; MAR/TNC 2006; HRI 2008, 2010, 2012, 2015), mientras que en el resto de la costa norte aún existe incertidumbre sobre la distribución y extensión de estos ecosistemas (Cortés 1997). Las referencias sobre la composición bentónica a lo largo de la plataforma continental son escasas, pero evidencian importantes coberturas arrecifales cercanas a Omoa (Cabrera 2010), en la Bahía de Tela (HRI 2012, 2015, Bodmer et al. 2015), en la Bahía de Trujillo (MAR/TNC 2006) y alrededor de los Cayos Miskitos (Chollett et al. 2013). Entre las costas de Tela y Trujillo se encuentra el Refugio de Vida Silvestre Barras de Cuero y Salado. Esta área protegida ha estado bajo manejo desde 1987, sin embargo, los administradores del área aún no tienen información sobre la distribución, la extensión y el estado de conservación de los arrecifes coralinos ubicados dentro de la misma. El conocimiento empírico de los pescadores artesanales locales y la composición de especies capturadas son hasta ahora la única guía.

Un conocimiento más detallado sobre los arrecifes coralinos en esta área protegida es importante ya que ahí hay comunidades que dependen de la pesca artesanal como principal actividad económica. De acuerdo al estudio de López (López 2008), las especies comerciales más importantes son el calale (Lutjanus synagris), la culila (Caranx crysos),

\footnotetext{
${ }^{1}$ Centro de Estudios Marinos, Colonia El Sauce, La Ceiba, Honduras. Correo electrónico: andres@estudiosmarinos.org

${ }^{2}$ Centro de Estudios Marinos, Colonia El Sauce, La Ceiba, Honduras. Correo electrónico: cristhian@estudiosmarinos.org
} 
el ronco (Haemulon plumieri), el yarano (Conodon nobilis) y el robalo (Centropomus undecimalis). En la pesca, los robalos están más asociados a hábitats lagunares costeros y las culilas a hábitats pelágicos, mientras que los calales y roncos son especies asociadas a los arrecifes coralinos.

Muchos peces que habitan los arrecifes coralinos muestran particionamiento de hábitats a través de su ciclo de vida (Jaxion-Harm et al. 2012). Por ejemplo, el calale requiere de bosques de manglar, de pastos marinos y de arrecifes de coral someros en distintas etapas de su ciclo de vida (Lieske y Myers 1994), mientras que el robalo migra entre los hábitats lagunares y fluviales bordeados de bosques de manglar y los pastos marinos, pero no se encuentra en los hábitats arrecifales (Fraser 1978).

Los hábitats lagunares bordeados de bosques de manglar se encuentran ampliamente distribuidos a lo largo del litoral norte de Honduras, donde muchas otras comunidades han dependido del robalo como un recurso pesquero de fácil acceso y sin necesidad de una flota de pesca sofisticada. No obstante, la falta de un manejo adecuado de estas pesquerías lagunares ha ocasionado su sobrexplotación y forzado a los pescadores, particularmente de las comunidades entre Tela y Salado Barra, a buscar fuentes alternativas de empleo o a invertir en equipo y barcos más grandes para pescar en mar abierto, transfiriendo así el esfuerzo de pesca hacia hábitats marinos adyacentes (Box 2012). De hecho, en el censo pesquero realizado por López (López 2008) en las principales comunidades pesqueras del Refugio de Vida Silvestre Barras de Cuero y Salado ya había una tendencia hacia más capturas de especies de pargos asociados a arrecifes coralinos, especialmente el calale.

El creciente desplazamiento del esfuerzo de pesca artesanal hacia hábitats arrecifales dentro de la plataforma continental como una reacción al colapso de las pesquerías en los sistemas lagunares costeros, requiere de un manejo efectivo para evitar un patrón de sobrexplotación. Bajo este esquema resulta importante tener información clara sobre la distribución y el estado de los hábitats que sustentan la producción pesquera local. Hay información técnica sobre los ecosistemas lacustres y fluviales a lo largo de la costa norte, incluido el refugio (Carrasco y Caviedes 2014). No obstante, de este aún no hay conocimiento comparable sobre sus hábitats bentónicos marinos.
Una idea general sobre la distribución potencial de los arrecifes coralinos fue provista en el 2005 por el Millennium Coral Reef Mapping Project (MCRMP) que utilizó imágenes satelitales con una resolución espacial de $30 \mathrm{~m}$ para mapear la cobertura de corales someros alrededor del mundo (Andréfouët et al. 2005). Sin embargo, los datos sobre la costa norte de Honduras corresponden a una clasificación de datos no validados y solo reflejan una distribución potencial de ecosistemas arrecifales. De acuerdo a esta proyección, no se visualiza una cobertura de coral significativa dentro del refugio y en este caso los arrecifes coralinos más cercanos serían los Bancos Salmedina a unos $10 \mathrm{~km}$ del límite norte del refugio.

Dentro de los límites del refugio existen áreas de hábitat arrecifal referidas como piedras por los pescadores artesanales locales que visitan estas áreas durante sus faenas. Además, la pesca cotidiana de pargos y roncos con línea y anzuelo evidencian la presencia de hábitats arrecifales, que si bien es cierto ya son utilizados por los pescadores desde hace mucho tiempo, son pocos los que se han sumergido para observar el hábitat bentónico en estas aguas generalmente turbias. Así, el conocimiento técnico sobre los arrecifes coralinos es pobre y no suficiente para dirigir acciones acertadas de manejo pesquero.

Uno de los principales factores que aluden a la productividad de un arrecife coralino y su capacidad para sostener la pesca a la que es sometido es su complejidad estructural. Este atributo físico puede caracterizarse de manera general a simple vista. Los arrecifes dominados por corales suaves (abanicos de mar), generalmente carecen de estructuras de alto relieve y tienden a ser sitios planos y sostienen una biomasa baja de peces. Por el contrario, los sitios con abundantes corales duros, por ejemplo del género Acropora, son hábitats tridimensionales altamente complejos con numerosas grietas y hendiduras que resultan ser importantes para la productividad pesquera. En la medida que la estructura del arrecife es más compleja, los organismos vulnerables, incluidos los peces juveniles y de talla pequeña, encuentran refugio contra la depredación.

Es bajo estas consideraciones que nuestro trabajo exploratorio tiene el objetivo de identificar de manera preliminar sitios de importancia para la productividad pesquera y priorizar sitios para una subsecuente caracterización biofísica detallada. 


\section{Materiales y Métodos}

Área de estudio. Se exploró el Refugio de Vida Silvestre Barras de Cuero y Salado y sus alrededores en la costa Caribe de Honduras (Figura 1). Esta es un área protegida compuesta por un complejo sistema fluvial $y$ de lagunas costeras entre las desembocaduras de los ríos Salado, Cuero y San Juan, además de una extensión marina. El área se ubica entre los $15^{\circ} 47^{\prime} 34^{\prime \prime}$ y $15^{\circ} 45^{\prime} 22^{\prime \prime}$ norte y $87^{\circ} 09^{\prime} 50^{\prime \prime}$ y $86^{\circ} 56^{\prime} 50^{\prime \prime}$ oeste aproximadamente a $30 \mathrm{~km}$ al oeste de la ciudad de La Ceiba y su polígono de extensión abarca 13,027 ha que incluye 5,037 ha de cobertura marina (ICF 2011).

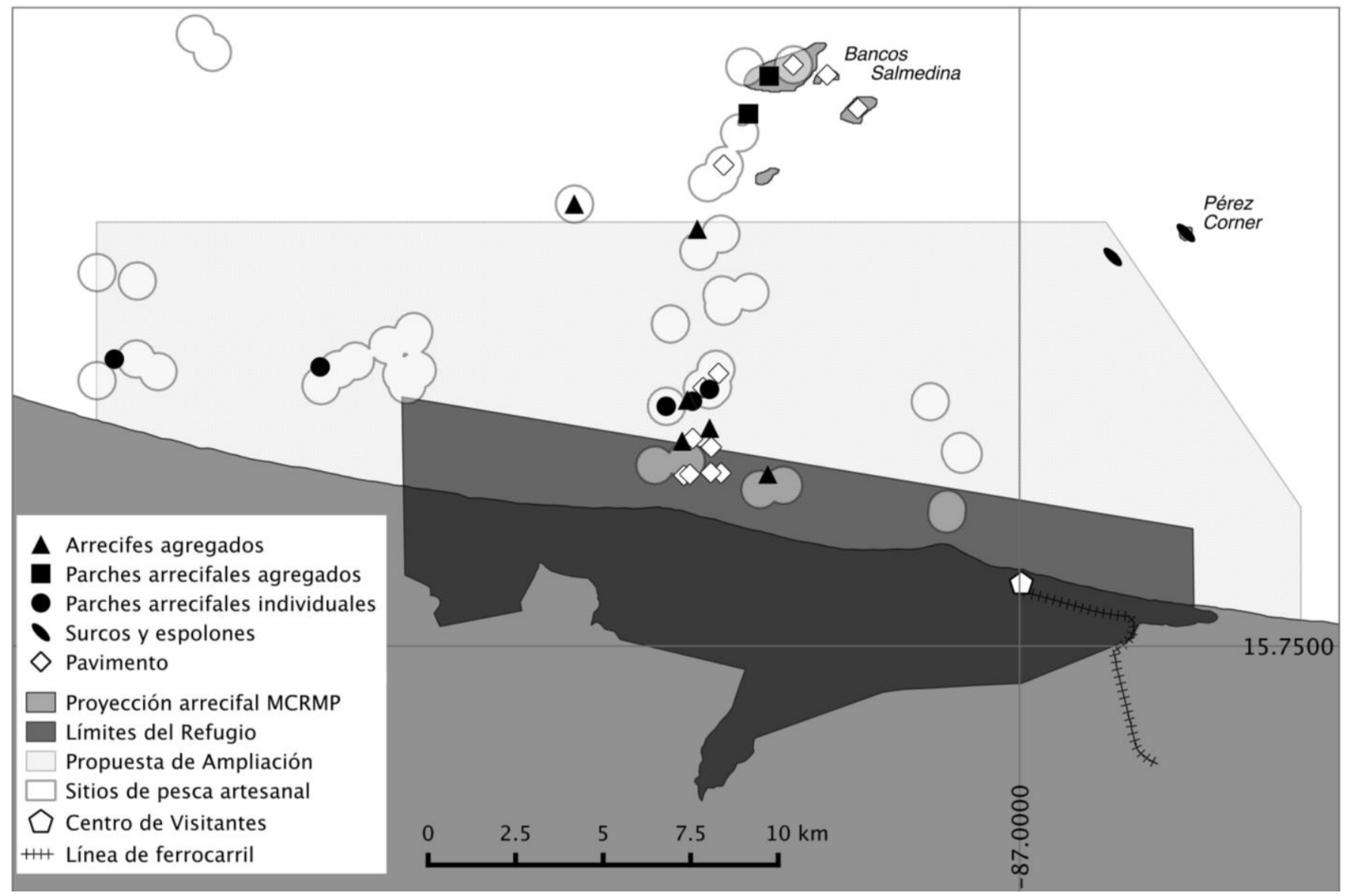

Figura 1. Distribución de arrecifes explorados dentro y alrededor del Refugio de Vida Silvestre Barras de Cuero y Salado, Honduras.

Se exploró el área marina dentro y alrededor de los límites del refugio teniendo como referencia un set de 51 sitios de pesca artesanal ya georreferenciados con pescadores locales. También se definieron sitios para confirmar la proyección del MCRP (Andréfouët et al. 2005). El trabajo de campo fue del 11 al 12 de agosto y del 11 al 14 de septiembre de 2015; y el 3 de febrero y el 3 de marzo de 2016. Las jornadas eran de máximo de 4 horas al día cada mañana, se exploraron puntos conocidos por el capitán de la embarcación más otros definidos al azar durante los trayectos.

Identificación de la cobertura bentónica. Se identificó la cobertura bentónica de tres formas 
complementarias: a) inmersión libre de un miembro del equipo para identificar visualmente el tipo de hábitat, observación de unos 5 minutos del bentos sobre un diámetro mínimo de $12 \mathrm{~m}$ en cada sitio, b) fotografía con cámara sumergible instalada sobre un ancla, c) inmersión con equipo SCUBA. Tras la identificación visual, la clasificación de bentos se basó en la utilizada por Zitello et al. (2009) bajo los atributos de estructura geomorfológica (e.g. arena o arrecife agregado) y cobertura biológica (e.g. coral vivo o pasto marino). Respecto a la estructura geomorfológica y considerando nuestra técnica de exploración in situ, definimos la unidad mínima de medición en $100 \mathrm{~m}^{2}$ a diferencia de $1,000 \mathrm{~m}^{2}$ que Zitello et al. (2009) definen para exploración remota.

Batimetría. En una embarcación abierta de 23 pies con motor de $40 \mathrm{HP}$ fuera de borda, se instaló un dispositivo Lowrance ${ }^{\circledR}$ StructureScan HD 8 para obtener lecturas batimétricas de 25 sitios explorados, así como durante los trayectos de un sitio a otro. Los datos fueron importados y procesados con el software ReefMaster $^{\circledR}$ v1.8. Durante las inmersiones en cada sitio se estimó superficialmente el grado de relieve distinguiéndolo como alto cuando habían arrecifes duros con alturas mayores a $1.5 \mathrm{~m}$, medio cuando oscilaban entre 0.5 y $1.5 \mathrm{~m}$ y bajo cuando la altura no superaba los $0.5 \mathrm{~m}$.

\section{Resultados}

Identificación de la cobertura bentónica. Se identificó la geomorfología y cobertura biológica en $22,800 \mathrm{~m}^{2}$ de hábitat submarino distribuido en 33 sitios (Figura 1, Cuadro 1). Siete sitios se ubican dentro de los límites del Refugio y 18 afuera de los límites oficiales, pero dentro de los límites según la iniciativa de ampliación (ICF 2012). Ocho sitios se encuentran afuera de ambos límites. En 15 sitios se encontraron arrecifes coralinos diferenciados en cuatro tipos de geomorfología: seis arrecifes agregados, cinco parches arrecifales individuales, dos parches arrecifales agregados y dos sitios con surcos $y$ espolones. Sobre los 18 sitios restantes se identificaron "arena" y "pavimento" como estructuras geomorfológicas.

Un arrecife agregado se refiere a una formación coralina continua, de alto relieve y de forma variable, pero que carece de canales de arena, surcos y espolones (Figura 2). Los parches arreficales individuales se encuentran aislados de otras formaciones coralinas por arena, pasto marino u otros hábitats; suelen ser de forma circular u oblicua y con un relieve mayor a $1 \mathrm{~m}$ (Zitello et al. 2009). Consideramos $100 \mathrm{~m}^{2}$ como tamaño mínimo de los parches arrecifales individuales. Los parches arrecifales agregados tienen las mismas características que los individuales, pero tienen menos de $100 \mathrm{~m}^{2}$ y están muy cerca unos de otros. Los surcos y espolones se refiere a la alternancia entre valles de arena y formaciones coralinas alargadas con un relieve alto. La arena se define como un sedimento grueso que se encuentra en zonas expuestas a corrientes 0 a la energía de las olas, mientras que el pavimento se refiere a una plataforma rocosa sólida, plana, de bajo relieve y cubierta por algas, corales, gorgonias u otros organismos sésiles que se encuentran densamente (Zitello et al. 2009).

De los seis sitios con arena como estructura geomorfológica, tres poseían algas como la cobertura biológica predominante y tres carecían de cobertura biológica. De los 15 sitios con arrecifes coralinos, ocho poseían algas como la cobertura biológica predominante y en siete una cobertura de coral vivo. Los siete sitios con coral vivo como cobertura biológica predominante son: Piedra El Pollo, Piedra Calale 2, Piedra Cubera 2, Piedra de Pablo, Piedra Culi, Flower's Garden y Salmedina 4.

En Piedra El Pollo hay una cobertura de coral lechuga (Undaria tenuifolia) con buena salud y poca cobertura de algas que se extiende por un diámetro no menor a $35 \mathrm{~m}$. También, en Piedra Calale 2 se observó una cobertura de Agaricia spp. con buena salud y poca cobertura de algas que se extendía por un diámetro no menor a $20 \mathrm{~m}$. En Piedra Cubera 1 hay una cobertura extensa de coral lechuga, misma que se desvanece al oeste para dar paso a un pavimento con abundantes abanicos de mar (Gorgonia spp.) que se extiende por $150 \mathrm{~m}$ hasta llegar a Piedra de Pablo. En este último sitio se observó arrecife agregado en un diámetro de unos $50 \mathrm{~m}$ donde prevalecen corales formadores de arrecife, particularmente Orbicella faveolata con alta cobertura de coral vivo. A unos $20 \mathrm{~m}$ al norte de este sitio se encuentra una caída de alrededor de $25 \mathrm{~m}$ paralela a la costa. Piedra Culi se encuentra a $33 \mathrm{~m}$ de profundidad y fue el arrecife coralino más profundo, tiene una dominancia de Agaricia spp. con un morfotipo platiodeo característico de sitios profundos, distribuida en un diámetro aproximado de $50 \mathrm{~m}$ sobre una planicie de bajo relieve, pero con ocasionales montículos de hasta $3 \mathrm{~m}$ 
Alegría y Pérez: Nuevos Reportes de Arrecifes Coralinos en el Caribe de Honduras

de altura. En Flower's Garden se encontraron surcos y espolones de alto relieve, hasta $4 \mathrm{~m}$ de altura, con una extensa cobertura de $U$. tenuifolia. Esta misma especie domina la cobertura del sitio Salmedina 4, que también presenta un alto relieve, pero distribuido en parches de arrecife individuales con diámetros menores a $10 \mathrm{~m}$ y separados por valles de arena.

Cuadro 1. Localización y atributos biofísicos de los sitios explorados (proyección WGS84) en el Refugio de Vida Silvestre Barras de Cuero y Salado (RVSBCS).

\begin{tabular}{|c|c|c|c|c|c|c|c|c|}
\hline Sitio & Latitud & Longitud & m & Geomorfología & Cobertura & Relieve & RVSBCS & $\begin{array}{c}\text { Área } \\
\text { Explorada } \\
\left(\mathrm{m}^{2}\right)\end{array}$ \\
\hline WP 52 & 15.8226 & -87.0801 & 18 & Pavimento & Algas & Bajo & Afuera $^{\S}$ & 100 \\
\hline Piedra 7 & 15.8493 & -87.0531 & 12 & Arena & & Bajo & Afuera & 100 \\
\hline Piedra Azope & 15.7993 & -87.0843 & 13 & Arena & & Bajo & Adentro & 100 \\
\hline Piedra Pluma & 15.7992 & -87.0888 & 12 & Arena & & Bajo & Adentro & 100 \\
\hline Piedra Ladío & 15.8444 & -87.0716 & 10 & Arena & Algas & Bajo & Afuera & 200 \\
\hline Famosa de Cuero & 15.8236 & -87.0810 & 18 & Arena & Algas & Bajo & Afuera & 100 \\
\hline WP 58 & 15.8181 & -87.0827 & 14 & Arena & Algas & Bajo & Afuera & 100 \\
\hline Piedra 8 & 15.8610 & -87.0858 & 11 & Arrecife agregado & Algas & Medio & Afuera & 400 \\
\hline Piedra Yuri & 15.8781 & -87.0787 & 14 & Pavimento & Algas & Medio & Afuera & 500 \\
\hline WP 45 & 15.7958 & -87.0670 & 11 & Arrecife agregado & Algas & Medio & Adentro & 200 \\
\hline WP 48 & 15.7957 & -87.0878 & 13 & Pavimento & Algas & Bajo & Adentro & 100 \\
\hline Piedra Seca & 15.8029 & -87.0821 & 12 & Pavimento & Algas & Bajo & Afuera & 100 \\
\hline Piedra Mota 1 & 15.8183 & -87.0826 & $s d^{*}$ & $\begin{array}{l}\text { Parche arrecifal } \\
\text { individual }\end{array}$ & Algas & Medio & Afuera & 100 \\
\hline Piedra Mota 2 & 15.8188 & -87.0842 & 17 & Pavimento & Algas & Bajo & Afuera & 200 \\
\hline Piedra de Pablo & 15.8155 & -87.0884 & 7 & Arrecife agregado & Coral vivo & Alto & Afuera & 2000 \\
\hline Piedra Cubera 1 & 15.8152 & -87.0871 & 14 & $\begin{array}{l}\text { Parche arrecifal } \\
\text { individual }\end{array}$ & Algas & Medio & Afuera & 500 \\
\hline Piedra Cubera 2 & 15.8138 & -87.0941 & 7 & $\begin{array}{l}\text { Parche arrecifal } \\
\text { individual }\end{array}$ & Coral vivo & Medio & Afuera & 500 \\
\hline Piedra El Pollo & 15.8046 & -87.0898 & 13 & Arrecife agregado & Coral vivo & Medio & Afuera & 1000 \\
\hline Piedra Calale 2 & 15.8080 & -87.0825 & 16 & Arrecife agregado & Coral vivo & Medio & Afuera & 300 \\
\hline Piedra Pejepluma & 15.8264 & -87.2412 & $\mathrm{sd}$ & $\begin{array}{l}\text { Parche arrecifal } \\
\text { individual }\end{array}$ & Algas & Medio & Afuera & 100 \\
\hline Piedra Culuco & 15.8243 & -87.1864 & $\mathrm{sd}$ & $\begin{array}{l}\text { Parche arrecifal } \\
\text { individual }\end{array}$ & Algas & Bajo & Afuera & 200 \\
\hline Piedra Víctor & 15.8052 & -87.0870 & 18 & Pavimento & Algas & Bajo & Afuera & 100 \\
\hline WP 47 & 15.7962 & -87.0822 & 11 & Pavimento & Algas & Bajo & Adentro & 100 \\
\hline WP 49 & 15.7954 & -87.0893 & 14 & Pavimento & Alqas & Bajo & Adentro & 100 \\
\hline WP 46 & 15.7960 & -87.0795 & 12 & Pavimento & Algas & Bajo & Adentro & 100 \\
\hline Piedra Culi & 15.8677 & -87.1186 & 33 & Arrecife agregado & Coral vivo & Bajo & Afuera & 2000 \\
\hline Perez Corner & 15.8600 & -86.9556 & 12 & $\begin{array}{l}\text { Surcos y } \\
\text { espolones }\end{array}$ & Algas & Medio & Afuera & 2000 \\
\hline Flower's Garden & 15.8536 & -86.9751 & 10 & $\begin{array}{l}\text { Surcos y } \\
\text { espolones }\end{array}$ & Coral vivo & Alto & Afuera & 1500 \\
\hline Salmedina 1 & 15.8932 & -87.0430 & 7 & Pavimento & Algas & Bajo & Afuera & 4500 \\
\hline Salmedina 2 & 15.9021 & -87.0512 & 7 & Pavimento & Algas & Bajo & Afuera & 2000 \\
\hline
\end{tabular}


Cuadro 1. Continuación

\begin{tabular}{|c|c|c|c|c|c|c|c|c|}
\hline Sitio & Latitud & Longitud & m & Geomorfología & Cobertura & Relieve & RVSBCS & $\begin{array}{c}\text { Área } \\
\text { Explorada } \\
\left(\mathrm{m}^{2}\right)\end{array}$ \\
\hline Salmedina 3 & 15.9048 & -87.0602 & 6 & Pavimento & Algas & Bajo & Afuera & 1500 \\
\hline Salmedina 4 & 15.9018 & -87.0666 & 12 & $\begin{array}{l}\text { Parches } \\
\text { arrecifales } \\
\text { agregados }\end{array}$ & Coral vivo & Alto & Afuera & 1000 \\
\hline Salmedina 5 & 15.8917 & -87.0722 & 12 & $\begin{array}{l}\text { Parches } \\
\text { arrecifales } \\
\text { agregados }\end{array}$ & Algas & Bajo & Afuera & 1000 \\
\hline
\end{tabular}

$\S=$ Oficialmente afuera del refugio, pero adentro del área de ampliación de acuerdo a iniciativa del ICF.

$¥$ = Sin datos.
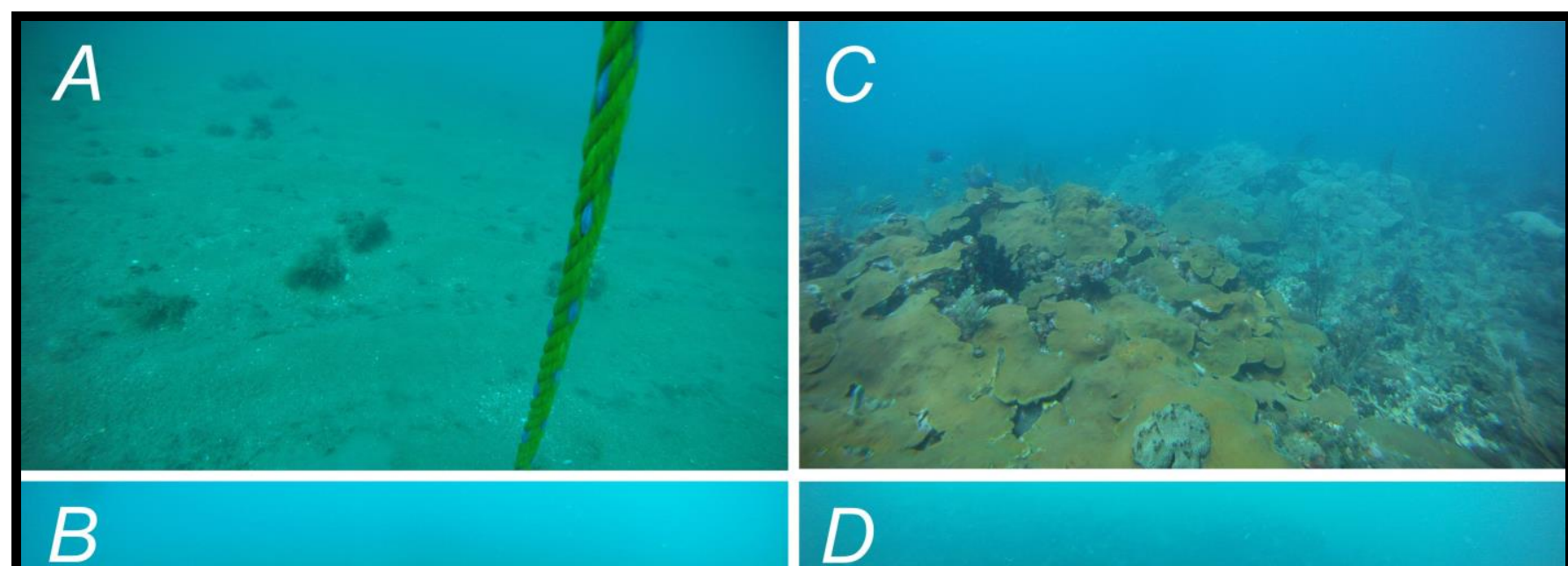

Figura 2. Refugio de Vida Silvestre Barras de Cuero y Salado, Honduras. A) Ejemplo de sedimentos no agregados (arena) sin cobertura biológica considerable en Piedra Pluma; B) Ejemplo de pavimento en Waypoint 47; C) Ejemplo de un arrecife agregado con colonias del coral masivo Orbicella faveolata en el sitio Piedra de Pablo; D) Ejemplo de surcos y espolones en Perez Corner. 
Batimetría. El rango de profundidad de los arrecifes coralinos varió entre 7 y $33 \mathrm{~m}$, mientras que la arena y el pavimento varió entre 6 y $18 \mathrm{~m}$. Veinte sitios presentaron un relieve bajo, diez sitios un relieve medio y tres sitios un relieve alto. Piedra Pablo fue uno de los sitios con alto relieve producto de una dominancia de corales hermatípicos masivos de la especie $O$. faveolata. El procesamiento de datos de sonar permitió la generación de polígonos batimétricos alrededor de cada sitio con extensión variable entre 0.5 y $2 \mathrm{~km}^{2}$ (Figura 3).

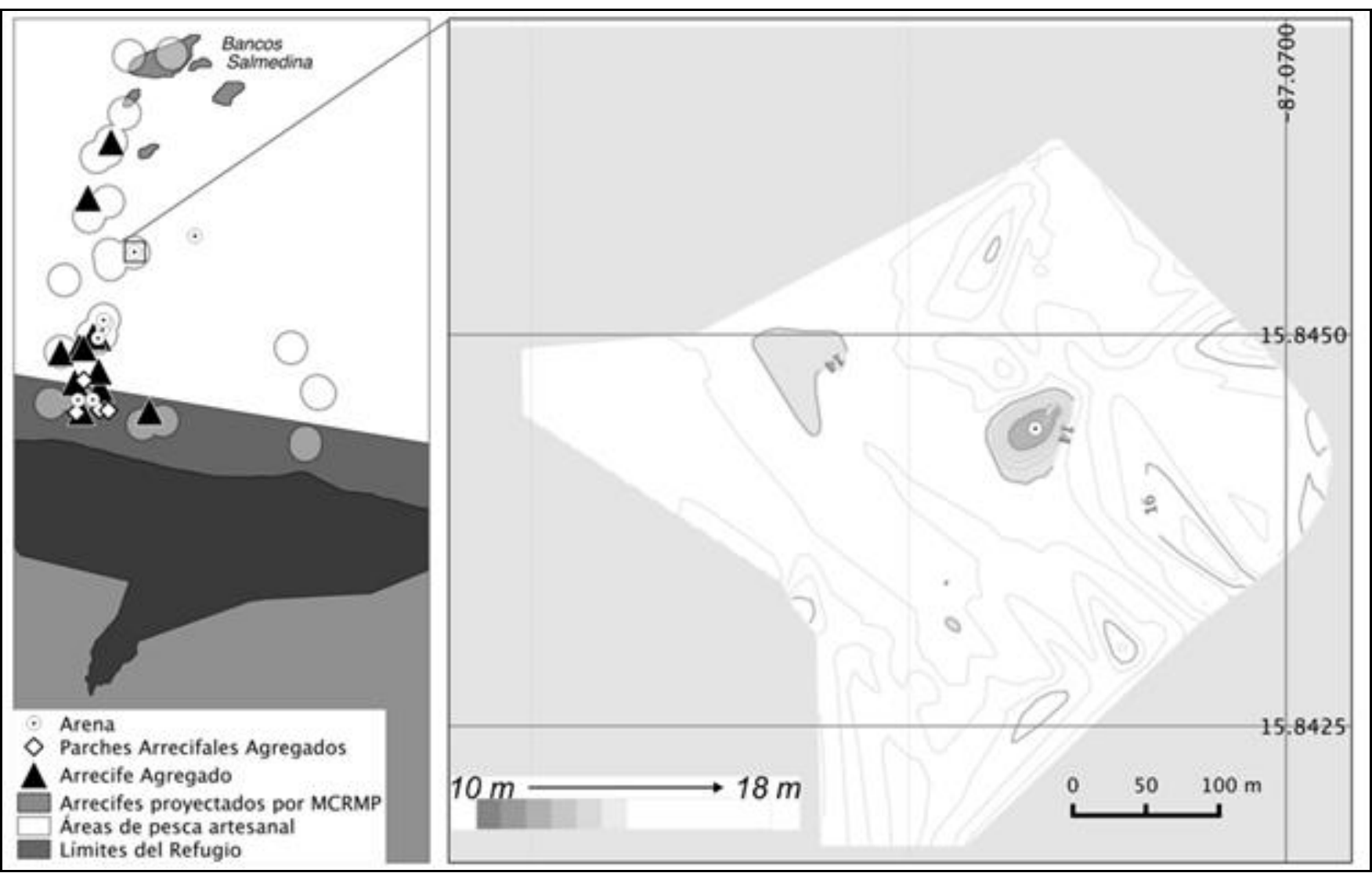

Figura 3. Mapa batimétrico sobre el sitio de pesca artesanal Piedra Ladío en el Refugio de Vida Silvestre Barras de Cuero y Salado, Honduras.

\section{Discusión}

Existen arrecifes coralinos con predominante cobertura de coral vivo a una distancia de $2-3 \mathrm{~km}$ al norte de la costa del Refugio de Vida Silvestre Barras de Cuero y Salado, pero fuera de sus límites marinos. Es de interés el sitio Piedra de Pablo donde dominan los corales hermatípicos masivos de O. faveolata, una especie de alta complejidad estructural y característica de arrecifes coralinos de alto valor ecosistémico y mayor biodiversidad (Mumby et al. 2008; Harborne 2009). Flower's Garden y Salmedina 4 son dos sitios con una complejidad estructural comparable a Piedra Pablo, pero con una cobertura menor de corales hermatípicos masivos. En los sitios El Pollo, Piedra Calale 2, Piedra Cubera 2 y Piedra Culi se encontraron 
coberturas amplias de coral vivo, sobretodo de Agaricia spp. y $U$. tenuifolia, que a pesar de tener más abundancia, contribuyen menos a la armazón del arrecife (Álvarez-Filip et al. 2009).

Ante la iniciativa de expansión limítrofe promovida por el ICF, resulta conveniente profundizar con una evaluación más detallada sobre la composición y salud de los arrecifes coralinos aquí reportados. También se propone expandir el esfuerzo de exploración para verificar otros arrecifes pronosticado por el MCRMP, particularmente el sector sur de Bancos Salmedina.

Los Bancos Salmedina, ubicados $11 \mathrm{~km}$ al norte del refugio, son sitios de pesca de los pescadores artesanales de Utila. De acuerdo a la proyección del MCRMP, estos se distribuyen en cinco bancos con una longitud máxima de 0.7 a $2.6 \mathrm{~km}$. En el banco del extremo este, a $350 \mathrm{~m}$ al oeste del sitio Salmedina 1, está el sitio UTI011 que fue evaluado en el 2006 bajo el protocolo del Atlantic and Gulf Rapid Reef Assessment (AGRRA) (MAR/TNC 2006). Los resultados atribuyeron a UTI011 una geomorfología de surcos y espolones con un relieve medio, una cobertura de coral vivo de $13.37 \%\left({ }^{+} / 10.29\right)$ dominada por U. tenuifolia y Siderastrea siderea. El porcentaje de coral vivo fue inferior al promedio caribeño de $16.3 \%$ al 2011 (Jackson et al. 2014), que sumado a la alta desviación estándar, la evaluación de UTI011 no es representativa de todo el sistema de Bancos Salmedina.

Perez Corner también fue evaluado en el 2006 bajo los mismos parámetros de UTI011, el sitio está dentro del área explorada por nosotros. Según la evaluación base, este sitio tiene una geomorfología de surcos y espolones con un relieve medio, una cobertura de coral vivo de $7.20 \%(+2.30)$ dominada por U. tenuifolia y Porites asteroides.

La determinación de evaluar UTI011 y Perez Corner en el 2006 estuvo basada en la proyección del MCRMP. Ambos sitios se caracterizaron por ser arrecifes coralinos en profundidades de $10 \mathrm{~m}$ y con una cobertura dominada por corales de baja complejidad estructural (MAR/TNC 2006). Con estas condiciones, el MCRMP identificó tales sitios, pero no a otros que aún con extensiones sobre los $1000 \mathrm{~m}^{2}$ y a pesar de ofrecer condiciones más favorables para la detección remota en términos de profundidad y relieve, por ejemplo Piedra de Pablo, no fueron detectados. Esta omisión pudo ser ocasionada por la turbidez del agua al tomar las imágenes o la forma de la estructura arrecifal.

Esta exploración es preliminar considerando que hay 24 sitios de pesca artesanal con georreferencia, pero aún no explorados. A excepción del WP 48, los sitios donde se encontró geomorfología de arena fueron referidos como sitios de pesca por los pescadores locales. Esto sugiere que la exploración se mantuvo al borde de una estructura arrecifal que pasó desapercibida debido al margen de error de la georreferencia.

Ante la evidencia de arrecifes coralinos dispersos y aún no reportados, surge la necesidad de ampliar el esfuerzo de exploración para crear un mapa completo sobre la distribución de arrecifes coralinos de extensión variable, pero de importancia para las producciones pesqueras locales. Para tal fin, se recomienda a las autoridades del Refugio de Vida Silvestre Barras de Cuero y Salado ampliar la exploración de hábitats bentónicos y considerar su distribución en la redefinición de límites marinos del área protegida.

Un aporte importante para guiar una nueva exploración, vendría de una encuesta cualitativa estructurada dirigida a pescadores locales para identificar y asignar un valor a los sitios de pesca respecto a su productividad pesquera actual e histórica. Mientras que la metodología utilizada en esta ocasión representa una manera expedita y de bajo costo para abordar el tema limítrofe, para fines de manejo pesquero será necesario seleccionar sitios clave para evaluaciones detalladas. Para ello se sugiere estimar el Índice Integrado de Salud Arrecifal (IISA) empleando el protocolo AGRRA, el cual requiere de inmersiones con equipo de buceo bajo condiciones climáticas que favorezcan la visibilidad y poca corriente, lo que generalmente ocurre durante el segundo trimestre del año. La selección de sitios para estudio a largo plazo, debe estar basada en un diseño comparativo entre áreas de pesca y áreas de no pesca.

Así mismo, se recomienda propiciar la protección explícita de los arrecifes ya identificados con prioridad a los arrecifes de alta y mediana complejidad. Los esfuerzos de manejo podrían enfocarse en impactos humanos tales como sedimentación y escorrentía de nutrientes, una acción de manejo más inmediata sería evitar su daño físico producto del anclaje por los pescadores. La instalación y el mantenimiento de 
boyas marcadas podrían reducir este tipo de daño directo.

Concluyentemente, se reconoce la importancia de documentar y evaluar la distribución de los arrecifes coralinos a lo largo de la costa norte de Honduras, para incorporarlos dentro de los esquemas de planificación para su uso sostenible. Así, y en concordancia con las recomendaciones dadas por Box (2012) tras una evaluación de las pesquerías artesanales de esta zona, durante los procesos de planificación se deberá contemplar la recuperación de las pesquerías en lagunas costeras y su uso rotativo con las pesquerías en arrecifes. El objetivo debe ser la diversificación de opciones de pesca y la distribución del esfuerzo entre estas dos zonas bajo un esquema de manejo que considere la conectividad entre estos dos espacios en términos ecológicos y de aprovechamiento.

Agradecimientos. Agradecemos el financiamiento por parte de La Red de Comunidades Turísticas de Honduras (LARECOTURH), y el apoyo de la Asociación de Pescadores Artesanales de La Rosita, Cuero y Salado (APROCUS), la Coral Reef Alliance (CORAL), la Fuerza Naval de Honduras (FNH) y la Fundación Cuero y Salado (FUCSA).

\section{Literatura Citada}

Álvarez-Filip, L., N. Dulvy, J. Gill, I. Côté y A. Watkinson. 2009. Flattening of Caribbean coral reefs: region-wide declines in architectural complexity. Proceedings of the Royal Society B: Biological Sciences 276(1669):30193025.

Andréfouët, S., F. Muller-Karger, J. Robinson, C. Kranenburg, D. Torres-Pulliza, S. Spraggins, y B. Murch. 2005. Global assessment of modern coral reef extent and diversity for regional science and management applications: a view from space. En Suzuki, Y., T. Nakamori, M. Hidaka, H. Kayanne, B. Casareto, K. Nadaoka, H. Yamano, M. Tsuchiya y K. Yamazato (eds). 10th International Coral Reef Symposium. Japanese Coral Reef Society, Okinawa, Japón.

Bodmer, M., A. Rogers, M. Speight, N. Lubbock y D. Exton. 2015. Using an isolated population boom to explore barriers to recovery in the keystone Caribbean coral reef herbivore Diadema antillarum. Coral Reefs 34:10111021.
Bouchon, S., L. Max, P. Portillo y W. Thompson. 2001. Los Ecosistemas Marinos y Costeros de las Islas de la Bahía. Proyecto Manejo Ambiental de las Islas de la Bahía - Subprograma Manejo Integral de Recursos Naturales (PMAIB). Consorcio Safege- SogreahMoncada y Moncada. Contrato HON/97/002/407. Reporte técnico. No. AMC 03.

Box, S. 2012. La pesca artesanal del Caribe Hondureño, desentrañando la pesca local compleja para identificar oportunidades de gestión. Agencia de los Estados Unidos para el Desarrollo Internacional (USAID) / Centro de Ecología Marina de Honduras, Honduras.

Cabrera, G. 2010. Diagnóstico Biofísico del Área Protegida Parque Nacional Cuyamel-Omoa (PANACO). ICF/PROCORREDOR. Honduras. $82 \mathrm{p}$.

Carrasco, J. y V. Caviedes. 2014. Diagnóstico de los Ecosistemas Marino - Costeros y de Agua Dulce de Honduras: Basado en Análisis de Viabilidad, Amenazas y Situación. ICF y USAID ProParque. $102 p$.

Chollett, I., S. Box, G. Stoyle. 2014. Honduran Miskito Cays: among the last unexplored reef systems in the Caribbean. Coral Reefs 33:155.

Fraser, T. 1978. Centropomidae. En W. Fischer (ed.) FAO species identification sheets for fishery purposes. West Atlantic (Fishing Area 31). FAO, Rome. Vol. 1-2. pag. var.

Harborne, A. 2009. First among equals: why some habitats should be considered more important than others during marine reserve planning. Environmental Conservation 36(2):87-90.

HRI. 2008. Libreta de calificaciones correspondiente al Sistema Arrecifal Mesoamericano: Una evaluación de la salud del ecosistema 2008. Healthy Reefs Initative. Disponible en www.healthyreefs.org.

HRI. 2010. Reporte de la Salud Ecológica del Arrecife Mesoamericano: Una evaluación de la salud del ecosistema 2010. Healthy Reefs Initative. Disponible en www.healthyreefs.org.

HRI. 2012. Reporte de la Salud Ecológica del Arrecife Mesoamericano: Una evaluación de la salud del ecosistema 2012. Healthy Reefs Initative. Disponible en www.healthyreefs.org.

HRI. 2015. Arrecife Mesoamericano una evaluación de la salud del ecosistema: Reporte del estado de salud 2015. Healthy Reefs Initative. Disponible en www.healthyreefs.org.

ICF. 2011. Plan de Manejo del Refugio de Vida Silvestre Barras de Cuero y Salado 2012 - 2016. Instituto Nacional de Conservación y Desarrollo Forestal, Áreas Protegidas y Vida Silvestre. Honduras. $208 \mathrm{p}$.

ICF. 2012. Acuerdo 030-2012 (Aviso de Ampliación del Refugio de Vida Silvestre Barras de Cuero y Salado). Instituto Nacional de Conservación y Desarrollo Forestal, Áreas Protegidas y Vida Silvestre. Honduras. 7 p. 
Jackson J., M. Donovan, K. Cramer y V. Lam (ed.). 2014. Status and Trends of Caribbean Coral Reefs: 19702012. Global Coral Reef Monitoring Network, IUCN, Gland, Switzerland. $304 \mathrm{p}$.

Jaxion-Harm, J., J. Saunders y M. Speight. 2012. Distribution of fish in seagrass, mangroves and coral reefs: life-stage dependent habitat use in Honduras. Revista Biología Tropical 60 (2): 683-698.

Lieske, E. y R. Myers. 1994. Collins Pocket Guide. Coral reef fishes. Indo-Pacific \& Caribbean including the Red Sea. Haper Collins Publishers. $400 \mathrm{p}$.

López, E. 2008. Diagnóstico de la pesca artesanal en el Refugio de Vida Silvestre Cuero y Salado. Informe de consultoría FUCSA-WWF. En: Rico, P. Medina, A. (eds). 2010. Plan de Manejo Pesquero del Refugio de Vida Silvestre Barras Cuero y Salado, Honduras (20102014). FUCSA/WWF/FFEM. 52 p.
MAR/TNC. 2006. Rapid Reef Assessment of Guatemala and Honduras sites in the MAR region towards the identification of bleaching resilient and resistant reefs. Mesoamerican Reef Proyect / The Nature Conservancy. $86 \mathrm{p}$.

Mumby, P., K. Broad, D. Brumbaugh, C. Dahlgren, A. Harborne, A. Hastings, K. Holmes, C. Kappel, F. Micheli y J. Sanchirico. 2008. Coral reef habitats as surrogates of species, ecological functions, and ecosystem services. Conservation Biology 22(4):941-951.

Zitello A., L. Bauer, T. Battista, P. Mueller, M. Kendall y M. Monaco. 2009. Shallow-Water Benthic Habitats of St. John, U.S. Virgin Islands. NOAA Technical Memorandum NOS NCCOS 96. Silver Spring, MD. 53 p.

Recibido para publicación el 15 de enero de 2016.

Aceptado para publicación el 16 de octubre de 2016 . 